REPRESENTATION THEORY

An Electronic Journal of the American Mathematical Society

Volume 13, Pages 63-81 (April 2, 2009)

S 1088-4165(09)00343-4

\title{
ON THE ASYMPTOTICS OF WHITTAKER FUNCTIONS
}

\author{
EREZ LAPID AND ZHENGYU MAO
}

\begin{abstract}
We study the asymptotics of Whittaker functions on split groups and relate them to the cuspidal exponents of the representation.
\end{abstract}

\section{INTRODUCTION}

Whittaker models are ubiquitous in the representation theory of quasi-split reductive groups over local fields. They comprise the bedrock for whole families of local zeta integrals of Rankin-Selberg type. In the analysis of the latter, it is imperative to know the asymptotics of Whittaker functions, in order to control the domain of convergence of the integrals. The basic example of $G L(2)$ was studied in detail in God70 (cf. also Example 3.7 below). More generally, this question was studied extensively in the literature, especially in the context of $G L_{n}$ (e.g. JS90b, JS90a, JPSS79, CPS]). In the Archimedean case a fairly complete answer is given in Wal92, Chapter 15]. On the other hand, to the best of the authors' knowledge, the exact connection between the asymptotics of the Whittaker functions and the exponents of the representation in the $p$-adic case is not made explicit in the literature. The purpose of this short note is to partially fill this gap. The precise statement is given in Theorem 3.1 below, and is motivated by the results above (cf. [JS90b, Proposition 2.2], JJS90a, §2], [JPSS79]). As a consequence, we realize the inner product of generic square-integrable and more generally, tempered representations, on the Whittaker model. For simplicity we work with split groups.

After completing an early version of this note we learned that Yiannis Sakellaridis and Akshay Venkatesh have launched an ambitious program to study the decomposition of the $L^{2}$-space of spherical varieties. In particular, they obtained asymptotic results of the kind which appear in this paper, in a very general setup. Although strictly speaking their current setup does not include the Whittaker case, there is no doubt that this can eventually be incorporated into the general scheme. In particular, Conjectures 3.5 and 3.8 below are probably within reach. Nevertheless, we believe that the Whittaker case is both sufficiently important and elementary to merit its own exposition.

Acknowledgement. We would like to thank Jim Cogdell, David Soudry, Yakov Varshavsky and Akshay Venkatesh for useful discussions. We are also very grateful to the referee for carefully reading this note, pointing out a few inaccuracies and making numerous suggestions.

Received by the editors July 23, 2008 and, in revised form, December 1, 2008.

2000 Mathematics Subject Classification. Primary 22E50, 11 F70.

This research partially funded by grant \#2004200 from the United States-Israel Binational Science Foundation. 


\section{Preliminaries}

2.1. Notation. Throughout, let $F$ be a non-archimedean local field of characteristic zero with valuation $v$ and normalized absolute value $|\cdot|=q^{-v(\cdot)}$. If $X$ is a variety over $F$, we will often denote its $F$-points by $X$ as well 11 It forms an $l$-space in the sense of BZ76. The space of compactly supported locally constant functions on $X$ will be denoted by $\mathcal{S}(X)$.

For any algebraic group $H$ over $F$ we denote its center by $Z_{H}$, its derived group by $H^{\text {der }}$ and the connected component of the identity element in $H$ by $H^{0}$. Let $X^{*}(H)$ be the lattice of rational characters of $H$ and set

$$
H^{1}=\bigcap_{\chi \in X^{*}(H)} \operatorname{Ker}|\chi|
$$

If $H$ is reductive then $Z_{H} H^{1}$ is of finite index in $H$. If $H$ is a subgroup of $G$, we denote its centralizer by $C_{G}(H)$.

If $T$ is a torus, then $T^{1}$ is the maximal compact subgroup of $T$. Suppose that $T$ is split. Then the lattice $X_{*}(T)$ of co-characters of $T$ can be identified with $\operatorname{Hom}\left(X^{*}(T), \mathbb{Z}\right)$ and the map

$$
H: T \rightarrow X_{*}(T)
$$

defined by

$$
H(\mu(a))=v(a) \mu, \mu \in X_{*}(T), a \in F^{*}
$$

induces an isomorphism

$$
T / T^{1} \rightarrow X_{*}(T)
$$

In other words,

$$
\langle\omega, H(t)\rangle=v(\omega(t))
$$

for all $t \in T$ and $\omega \in X^{*}(T)$. Any $\lambda \in X^{*}(T) \otimes_{\mathbb{Z}} \mathbb{R}$ defines a character

$$
|\lambda|: T \rightarrow \mathbb{R}_{+}
$$

satisfying

$$
|\lambda|(\mu(a))=|a|^{\langle\lambda, \mu\rangle}
$$

for any $\mu \in X_{*}(T), a \in F^{*}$. Moreover, any continuous character $T \rightarrow \mathbb{R}_{+}$arises this way for a unique $\lambda \in X^{*}(T) \otimes_{\mathbb{Z}} \mathbb{R}$. In particular, to any continuous character

$$
\chi: T \rightarrow \mathbb{C}^{*}
$$

we can attach

$$
\operatorname{Re} \chi \in X^{*}(T) \otimes_{\mathbb{Z}} \mathbb{R}
$$

such that

$$
|\chi(t)|=|\operatorname{Re} \chi|(t)
$$

for all $t \in T$. Similarly, any $\lambda \in X^{*}(T) \otimes_{\mathbb{Z}} \mathbb{C}$ defines a character

$$
T / T^{1} \rightarrow \mathbb{C}^{*}
$$

by the formula

$$
t \mapsto q^{-\langle\lambda, H(t)\rangle} .
$$

All characters of $T / T^{1}$ are of this form and $\lambda$ is uniquely determined modulo $\frac{2 \pi \mathrm{i}}{\log q} X^{*}(T)$.

\footnotetext{
${ }^{1}$ An unfortunate outcome of this convention is the possible ambiguity in the notation $H \backslash G$ when $G$ and $H$ are groups over $F$. However, in this paper such quotients show up only when the first Galois cohomology group of $H$ is trivial, in which case there is no ambiguity.
} 
We say that a function $f: H \rightarrow \mathbb{C}$ is smooth if it is invariant under right translation by an open subgroup of $H$. We denote the space of smooth functions on $H$ by $C^{\infty}(H)$ and by $R$ the right regular representation of $H$ on $C^{\infty}(H)$.

Throughout, $G$ will be a connected reductive group which is split over $F$. Fix a Borel subgroup $B$ of $G$ and a maximal torus $T_{0}$ contained in $B$, both defined over $F$. Let $U_{0}$ be the unipotent radical of $B$, so that $B=T_{0} U_{0}$. We choose a maximal compact $K$ of $G$ in good position with respect to $T_{0}$. In particular, $G=B K$.

\subsection{Roots and weights. We set}

$$
\mathfrak{a}_{0}^{*}=X^{*}\left(T_{0}\right) \otimes_{\mathbb{Z}} \mathbb{R}, \quad \mathfrak{a}_{0}=X_{*}\left(T_{0}\right) \otimes_{\mathbb{Z}} \mathbb{R}
$$

with the canonical pairing. Let

$$
\Delta_{0} \subseteq X^{*}\left(T_{0}\right) \subseteq \mathfrak{a}_{0}^{*}
$$

denote the set of simple roots of $T_{0}$ on $\operatorname{Lie}\left(U_{0}\right)$. For each $\alpha \in \Delta_{0}$ let $U_{\alpha}$ be the oneparameter unipotent subgroup corresponding to $\alpha$. We extend $H: T_{0} \rightarrow X_{*}\left(T_{0}\right)$ to $G$ by requiring that $H$ is left- $U_{0}$ and right- $K$ invariant.

Henceforth, $P=M U$ will always denote a parabolic subgroup containing $B, U$ its unipotent radical and $M$ its Levi part containing $T_{0}$. Similarly, for $P^{\prime}=M^{\prime} U^{\prime}$, etc. The simple root $\Delta_{0}^{P}$ of $T_{0}$ on $\operatorname{Lie}\left(U_{0} \cap M\right)$ is a subset of $\Delta_{0}$. The set $\Delta_{0}^{P}$ determines $P$ uniquely and any subset of $\Delta_{0}$ arises in this way. The torus

$$
T_{0}^{M}=T_{0} \cap M^{\text {der }}
$$

is a maximal (split) torus in $M^{\text {der }}$ and we have

$$
Z_{M}=\bigcap_{\chi \in \Delta_{0}^{P}} \operatorname{Ker} \chi
$$

Setting

$$
T_{M}=Z_{M}^{0}
$$

we have $M=C_{G}\left(T_{M}\right)$. Moreover, $M / T_{M} M^{1}$ is a finite abelian group. We write

$$
\mathfrak{a}_{M}^{*}=X^{*}\left(T_{M}\right) \otimes_{\mathbb{Z}} \mathbb{R}
$$

and

$$
\left(\mathfrak{a}_{0}^{M}\right)^{*}=X^{*}\left(T_{0}^{M}\right) \otimes_{\mathbb{Z}} \mathbb{R} .
$$

Let $\mathfrak{a}_{M}$ and $\mathfrak{a}_{0}^{M}$ be the respective dual spaces. The set $\Delta_{0}^{P}$ is a basis for $\left(\mathfrak{a}_{0}^{M}\right)^{*}$ and the restriction maps

$$
X^{*}\left(T_{0}\right) \rightarrow X^{*}\left(T_{M}\right), \quad X^{*}\left(T_{0}\right) \rightarrow X^{*}\left(T_{0}^{M}\right)
$$

induce the isomorphisms

$$
\mathfrak{a}_{0}^{*}=\mathfrak{a}_{M}^{*} \oplus\left(\mathfrak{a}_{0}^{M}\right)^{*}, \quad \mathfrak{a}_{0}=\mathfrak{a}_{M} \oplus \mathfrak{a}_{0}^{M} .
$$

Similarly, the restriction map

$$
X^{*}(M) \rightarrow X^{*}\left(T_{M}\right)
$$

induces an isomorphism

$$
\mathfrak{a}_{M}^{*}=X^{*}(M) \otimes_{\mathbb{Z}} \mathbb{R} .
$$

Thus, any character $\chi: T_{M} \rightarrow \mathbb{R}_{+}$uniquely extends to $M$.

We let

$$
\hat{\Delta}_{0}^{\vee}=\left\{\varpi_{\alpha}^{\vee}: \alpha \in \Delta_{0}\right\}
$$


be the dual basis of $\Delta_{0}$ in $\mathfrak{a}_{0}^{G}$. Let

$$
\Delta_{P} \subseteq X^{*}\left(T_{M}\right) \subseteq \mathfrak{a}_{M}^{*}
$$

be the set of "simple roots" of $T_{M}$ on $U$, i.e., the restrictions of the roots $\Delta_{0} \backslash \Delta_{0}^{P}$ to $T_{M}$. Then

$$
\hat{\Delta}_{P}^{\vee}=\hat{\Delta}_{0}^{\vee} \cap \mathfrak{a}_{M}
$$

is a dual basis of $\Delta_{P}$ in $\mathfrak{a}_{M}^{G}=\mathfrak{a}_{M} \cap \mathfrak{a}_{0}^{G}$.

Remark 2.1. Suppose that $\alpha \in \Delta_{P}$ is the restriction of $\beta \in \Delta_{0} \backslash \Delta_{0}^{P}$. Then in $\mathfrak{a}_{0}^{*}$ we have

$$
\alpha=\beta+\sum_{\gamma \in \Delta_{0}^{P}} m_{\gamma} \gamma
$$

where $m_{\gamma} \geq 0$ for all $\gamma \in \Delta_{0}^{P}$.

Indeed, let $\lambda=\alpha-\beta \in\left(\mathfrak{a}_{0}^{M}\right)^{*}$. Then for any $\gamma \in \Delta_{0}^{P}$, if $\gamma^{\vee} \in X_{*}\left(T_{0}\right)$ denotes the corresponding co-root, then

$$
0=\left\langle\alpha, \gamma^{\vee}\right\rangle=\left\langle\beta, \gamma^{\vee}\right\rangle+\left\langle\lambda, \gamma^{\vee}\right\rangle \leq\left\langle\lambda, \gamma^{\vee}\right\rangle \text {. }
$$

Therefore, $\lambda$ is in the closure of the positive Weyl chamber of $\left(\mathfrak{a}_{0}^{M}\right)^{*}$, and in particular, it is a non-negative linear combination of $\Delta_{0}^{P}$.

If $P_{i} \supseteq B, i=1,2$, we will denote by $P_{1} \bullet P_{2}$ the parabolic subgroup generated by $P_{1}$ and $P_{2}$. Its Levi subgroup $M_{1} \bullet M_{2}$ is the group generated by $M_{1}$ and $M_{2}$. We have

$$
\begin{aligned}
Z_{M_{1} \bullet M_{2}} & =Z_{M_{1}} \cap Z_{M_{2}}, \\
\Delta_{0}^{P_{1} \bullet P_{2}} & =\Delta_{0}^{P_{1}} \cup \Delta_{0}^{P_{2}}, \\
\hat{\Delta}_{P_{1} \bullet P_{2}}^{\vee} & =\hat{\Delta}_{P_{1}}^{\vee} \cap \hat{\Delta}_{P_{2}}^{\vee}, \\
\mathfrak{a}_{0}^{P_{1} \bullet P_{2}} & =\mathfrak{a}_{0}^{P_{1}}+\mathfrak{a}_{0}^{P_{2}}, \\
\mathfrak{a}_{P_{1} \bullet P_{2}} & =\mathfrak{a}_{P_{1}} \cap \mathfrak{a}_{P_{2}},
\end{aligned}
$$

and similarly for the dual spaces.

We denote by $\delta_{P}$ the modulus function of $P$, viewed as a character of $M$. Observe that if $P^{\prime} \subseteq P$, then

$$
\delta_{P^{\prime}} \equiv \delta_{P} \text { on } Z_{M}
$$

\subsection{Eventually polynomial exponential sequences.}

Definition 2.2. A sequence $a_{n}, n \in \mathbb{Z}$ of complex numbers is called eventually polynomial exponential (e.p.e.) if there exists a finite subset $\Lambda$ of $\mathbb{C} / \frac{2 \pi i}{\log q} \mathbb{Z}$ and polynomials $P_{\lambda}, \lambda \in \Lambda$ such that $a_{n}=0$ for $n \ll 0$ and

$$
a_{n}=\sum_{\lambda \in \Lambda} P_{\lambda}(n) q^{\lambda n}
$$

for $n \gg 0$.

Clearly, for an e.p.e. sequence as above, the set $\Lambda$ is uniquely determined provided that $P_{\lambda}$ is non-zero for all $\lambda \in \Lambda$. We call $\Lambda$ the set of exponents of the sequence $a_{n}$ and denote it by $\mathcal{E}\left(\left(a_{n}\right)\right)$. 
The $q$-transform $Q\left(\left(a_{n}\right), s\right)$ of a sequence $a_{n}$ is by definition the formal power series

$$
\sum_{n \in \mathbb{Z}} a_{n} q^{-n s} .
$$

If $T$ is the right shift operation on sequences, then

$$
Q\left(T\left(\left(a_{n}\right)\right), s\right)=q^{s} Q\left(\left(a_{n}\right), s\right) .
$$

The following lemma is elementary.

Lemma 2.3. Let $a_{n}$ be a sequence of complex numbers such that $a_{n}=0$ for $n \ll 0$ and $\Lambda \subseteq \mathbb{C} / \frac{2 \pi \mathrm{i}}{\log q} \mathbb{Z}$ be a finite set. The following conditions are equivalent:

(1) $a_{n}$ is e.p.e. with $\mathcal{E}\left(\left(a_{n}\right)\right) \subseteq \Lambda$.

(2) There exists $m \geq 0$ such that the sequence

$$
\prod_{\lambda \in \Lambda}\left(T-q^{\lambda}\right)^{m}\left(a_{n}\right)
$$

has only finitely many non-zero terms.

(3) $Q\left(\left(a_{n}\right), s\right)$ converges absolutely for $\operatorname{Re} s>\max _{\Lambda} \operatorname{Re} \lambda$ and extends to a rational function in $q^{s}$ whose poles are contained in

$$
\left\{s \in \mathbb{C}: q^{s} \in q^{\Lambda}\right\} .
$$

Moreover, if $a_{n}$ has the form (2.3) and $q^{s_{0}}=q^{\lambda}, \lambda \in \Lambda$, then $Q\left(\left(a_{n}\right), s\right)$ admits a pole of order $d+1$ at $s_{0}$ where $d=\operatorname{deg} P_{\lambda}$ and the leading coefficient in the Laurent expansion of $Q\left(\left(a_{n}\right), s\right)$ around $s_{0}$ is $\frac{d !}{(\log q)^{d+1}} b$ where $b$ is the leading coefficient of $P_{\lambda}$.

Proof. It is easy to see that the first condition implies the second. The converse direction reduces by induction to the following statement. If $a_{n}=0$ for $n \ll 0$ and

$$
b_{n}=\left(T-q^{\lambda}\right) a_{n}
$$

is e.p.e., then $a_{n}$ is e.p.e. and

$$
\exp \left(\left(a_{n}\right)\right) \subseteq \exp \left(\left(b_{n}\right)\right) \cup\{\lambda\}
$$

To show this, note that

$$
a_{n}=\sum_{m=0}^{\infty} q^{\lambda m} b_{n-m-1}
$$

where only finitely many terms are non-zero in the sum. It is enough to consider the case where

$$
b_{n}= \begin{cases}Q(n) q^{\mu n} & \text { if } n \geq 0, \\ 0 & \text { otherwise }\end{cases}
$$

for some polynomial $Q$ and $\mu \in \mathbb{C}$. By writing $Q(x)$ as a linear combination of Newton polynomials $\left(\begin{array}{l}x \\ k\end{array}\right)$, the assertion now follows from the identity

$$
\sum_{n=0}^{m}\left(\begin{array}{l}
n \\
k
\end{array}\right) x^{n}=\frac{x^{k}}{k !}\left[\frac{1-x^{m+1}}{1-x}\right]^{(k)}, x \neq 1 .
$$

To show that the first condition implies the third we express each $P_{\lambda}$ as a linear combination of Newton polynomials and use the identity

$$
\sum_{n=0}^{\infty}\left(\begin{array}{l}
n \\
k
\end{array}\right) x^{n}=x^{k}(1-x)^{-(k+1)},|x|<1 .
$$

This also shows the very last part of the lemma. 
Finally, suppose that $Q\left(\left(a_{n}\right), s\right)$ satisfies condition (3). Then for some $m$,

$$
\left[\prod_{\lambda \in \Lambda}\left(q^{s}-q^{\lambda}\right)^{m}\right] Q\left(\left(a_{n}\right), s\right)
$$

is a rational function in $q^{s}$ without poles, and hence a Laurent polynomial in $q^{s}$. This implies the second condition by (2.4).

Corollary 2.4. Suppose that $a_{n}=0$ for $n \ll 0$ and there exists a positive integer $h$ and $\lambda \in \mathbb{C} / \frac{2 \pi \mathrm{i}}{\log q} \mathbb{Z}$ such that $b_{n}=a_{n+h}-q^{\lambda} a_{n}$ is e.p.e. Then $a_{n}$ is e.p.e. and

$$
\mathcal{E}\left(\left(a_{n}\right)\right) \subseteq \mathcal{E}\left(\left(b_{n}\right)\right) \cup\left\{\frac{\lambda+\frac{2 \pi \mathrm{i}}{\log q} j}{h}, 0 \leq j<h\right\} .
$$

In particular,

$$
\left\{\left|q^{\mu}\right|: \mu \in \mathcal{E}\left(\left(a_{n}\right)\right)\right\} \subseteq\left\{\left|q^{\mu}\right|: \mu \in \mathcal{E}\left(\left(b_{n}\right)\right)\right\} \cup\left\{q^{\operatorname{Re} \lambda / h}\right\} .
$$

In the case of a sequence with non-negative real numbers we can say a little more.

Lemma 2.5. Suppose that the sequence $a_{n}, n \in \mathbb{Z}$ is e.p.e. and $a_{n} \geq 0$ for all n. Let $\Lambda$ and $P_{\lambda}$ be as in (2.3) with $P_{\lambda} \neq \equiv 0$ for all $\lambda \in \Lambda$. Suppose further that $\operatorname{Re} \lambda \leq 0$ for all $\lambda \in \Lambda$. Then either $0 \notin \Lambda$, in which case $\operatorname{Re} \lambda<0$ for all $\lambda \in \Lambda$, $a_{n}$ is rapidly decreasing, and

$$
\sum_{n \in \mathbb{Z}} a_{n}=Q\left(\left(a_{n}\right), 0\right)
$$

or else we have:

(1) The highest coefficient of $P_{0}$ is positive.

(2) $\operatorname{deg} P_{\lambda} \leq d:=\operatorname{deg} P_{0}$ for all $\lambda \in \Lambda^{*}:=\Lambda \cap \mathrm{i} \mathbb{R} / \frac{2 \pi \mathrm{i}}{\log q} \mathbb{Z}$.

(3) $a_{n}=O\left(n^{d}\right)$.

(4) $Q\left(\left(a_{n}\right), s\right)$ converges for $\operatorname{Re} s>0$ and has a pole of order $r=d+1$ at $s=0$. Let $\kappa=\lim _{s \rightarrow 0} s^{r} Q\left(\left(a_{n}\right), s\right)$ be the leading coefficient. Then

$$
\sum_{n \leq m} a_{n} \sim \frac{(\log q)^{r} \kappa}{r !} m^{r} \quad \text { as } m \rightarrow \infty
$$

Proof. The lemma is straightforward if $\operatorname{Re} \lambda<0$ for all $\lambda \in \Lambda$, i.e., if $\Lambda^{*}=\emptyset$. Assume that $\Lambda^{*} \neq \emptyset$ and let $l=\max _{\lambda \in \Lambda^{*}} \operatorname{deg} P_{\lambda} \geq 0$. Let $h_{\lambda}$ be the coefficient of $x^{l}$ in $P_{\lambda}$. We have

$$
b_{n}:=\sum_{\lambda \in \Lambda^{*}} h_{\lambda} q^{\lambda n}=c_{n}+d_{n}
$$

where $c_{n}=\frac{a_{n}}{n^{t}} \geq 0$ and $d_{n}=O\left(\frac{1}{n}\right)$ as $n \rightarrow \infty$. For $m \geq 0$ consider

$$
x_{m}=\frac{1}{m} \sum_{n=1}^{m} b_{n}, \quad y_{m}=\frac{1}{m} \sum_{n=1}^{m}\left|b_{n}\right|, \quad z_{m}=\frac{1}{m} \sum_{n=1}^{m}\left|b_{n}\right|^{2} .
$$

By summing the geometric series we get

$$
\left|x_{m}-h_{0}\right|=o(1)
$$


By (2.6),

$$
\left|x_{m}-y_{m}\right| \leq \frac{1}{m} \sum_{n=1}^{m}\left|d_{n}\right|=o(1)
$$

Therefore,

$$
\left|y_{m}-h_{0}\right|=o(1)
$$

On the other hand,

$$
\left|b_{n}\right|^{2}=\sum_{\lambda, \lambda^{\prime} \in \Lambda^{*}} h_{\lambda} \overline{h_{\lambda^{\prime}}} q^{\left(\lambda-\lambda^{\prime}\right) n}
$$

so that

$$
\left|z_{m}-H\right|=o(1)
$$

where

$$
H=\sum_{\lambda \in \Lambda^{*}}\left|h_{\lambda}\right|^{2}>0
$$

Since $b_{n}$ is bounded, $z_{m}$ is bounded by a constant multiple of $y_{m}$. From (2.7) and (2.8) we conclude that $h_{0}>0$ so that $\operatorname{deg} P_{0}=l$. The first three parts of the lemma follow immediately.

Observe that for any polynomial $P$ and $\lambda \in \mathbb{C} \backslash \frac{2 \pi \mathrm{i}}{\log q} \mathbb{Z}$ with $\operatorname{Re} \lambda \leq 0$ we have

$$
\left|\sum_{n=0}^{m} P(n) q^{\lambda n}\right|= \begin{cases}O\left(m^{\operatorname{deg} P}\right) & \text { if } \operatorname{Re} \lambda=0, \\ O(1) & \text { if } \operatorname{Re} \lambda<0,\end{cases}
$$

as $m \rightarrow \infty$. Indeed, this follows from (2.5) by decomposing $P$ into Newton polynomials. On the other hand, by the last part of Lemma 2.3 we have

$$
\frac{(\log q)^{r}}{r !} \kappa=\frac{h_{0}}{r}
$$

Therefore, the last part of the lemma reduces to the relation

$$
\sum_{n=0}^{m} P_{0}(n) \sim \frac{h_{0}}{r} m^{r} \quad \text { as } m \rightarrow \infty .
$$

By ignoring lower order terms in $P_{0}$ we can assume that $P_{0}(n)=\left(\begin{array}{l}n \\ d\end{array}\right)$. It remains to apply the identity

$$
\sum_{n=0}^{m}\left(\begin{array}{l}
n \\
d
\end{array}\right)=\left(\begin{array}{c}
m+1 \\
d+1
\end{array}\right)
$$

2.4. A space of functions on $T_{0}$. Let $V$ be a representation space of a locally compact abelian group $A$. For any character $\chi$ of $A$ and $n \in \mathbb{N}$ we write

$$
V_{\chi, n}=\left\{u \in V:\left(a_{1}-\chi\left(a_{1}\right)\right) \ldots\left(a_{n}-\chi\left(a_{n}\right)\right) u=0 \quad \text { for all } a_{1}, \ldots, a_{n} \in A\right\} .
$$

We also denote by

$$
V_{\chi}=\bigcup_{n=0}^{\infty} V_{\chi, n}
$$

the generalized $\chi$-eigenspace and by

$$
V_{A-\text { fin }}=\bigoplus_{\chi \in \hat{A}} V_{\chi}
$$


the space of $A$-finite vectors in $V$. In particular, for $V=$ functions on $A$, we write

$$
\mathcal{F}(A)=V_{A \text {-fin }}
$$

for the space of $A$-finite functions on $A$. Note that for any $\chi_{i} \in \hat{A}, i=1,2$,

$$
\mathcal{F}(A)_{\chi_{1}} \mathcal{F}(A)_{\chi_{2}} \subseteq \mathcal{F}(A)_{\chi_{1} \chi_{2}} .
$$

For example, if $A=\mathbb{Z}^{r}$, then $\mathcal{F}(A)$ is the space of polynomial exponential functions on $A$. Similarly, if $T$ is a split torus and $\chi \in \hat{T}$, then it is easy to see by induction on $n$ that

$$
\mathcal{F}(T)_{\chi, n}=\{\chi(t) Q(H(t))\}
$$

where $Q$ ranges over the polynomials on $X_{*}(T)$ of degree $<n$.

We consider the affine line $\mathbb{A}^{1}$ as a monoid with respect to multiplication. The multiplicative group $\mathbb{G}_{m}$ is an open subgroup of $\mathbb{A}^{1}$. For any parabolic subgroup $P$ we consider the monoids

$$
\mathbb{M}_{P}=\prod_{\alpha \in \Delta_{0}} \begin{cases}\mathbb{G}_{m} & \text { if } \alpha \in \Delta_{0}^{P} \\ \mathbb{A}^{1} & \text { otherwise }\end{cases}
$$

In particular,

$$
\mathbb{M}_{G}=\mathbb{G}_{m}^{\Delta_{0}} .
$$

For any $P^{\prime} \subseteq P, \mathbb{M}_{P}$ is an open submonoid of $\mathbb{M}_{P^{\prime}}$. We view $\mathcal{S}\left(\mathbb{M}_{P}\right)$ as the ideal of $\mathcal{S}\left(\mathbb{M}_{P^{\prime}}\right)$ (under pointwise multiplication) consisting of those functions which are supported in $\mathbb{M}_{P}$, that is, vanish on $\mathbb{M}_{P^{\prime}} \backslash \mathbb{M}_{P}$. More generally, we have

$$
\mathcal{S}\left(\mathbb{M}_{P_{1}}\right) \mathcal{S}\left(\mathbb{M}_{P_{2}}\right) \subseteq \mathcal{S}\left(\mathbb{M}_{P_{1} \bullet P_{2}}\right)
$$

for any $P_{1}, P_{2}$. Each space $\mathcal{S}\left(\mathbb{M}_{P}\right)$ is invariant under right translation by $\mathbb{M}_{G}$. Let

$$
\mathfrak{r}: T_{0} \rightarrow \mathbb{M}_{G}
$$

be the homomorphism defined by

$$
\mathfrak{r}(t)_{\alpha}=\alpha(t)
$$

Note that by (2.1) we have

$$
\text { Ker } \mathfrak{r}=Z_{G} .
$$

For any $P$ and $\chi \in \widehat{Z_{M}}$, we denote by

$$
\mathfrak{F}_{P, \chi}=\mathfrak{F}_{P, \chi}^{G}
$$

(resp. $\mathfrak{F}_{P, \chi, n}$ ) the subspace of $C^{\infty}\left(T_{0}\right)$ spanned by functions of the form

$$
\xi(t) \varphi(\mathfrak{r}(t))
$$

where $\xi \in \mathcal{F}\left(T_{0}\right)_{\chi}$ (resp. $\left.\xi \in \mathcal{F}\left(T_{0}\right)_{\chi, n}\right)$ and $\varphi \in \mathcal{S}\left(\mathbb{M}_{P}\right)$. Recall that by our convention $\mathcal{F}\left(T_{0}\right)_{\chi}$ is the space of $T_{0}$-finite functions on $T_{0}$ which are generalized $\left(Z_{M}, \chi\right)$-eigenfunctions. Alternatively, we have

$$
\mathcal{F}\left(T_{0}\right)_{\chi}=\sum_{\chi^{\prime} \in \widehat{T_{0}}:\left.\chi^{\prime}\right|_{Z_{M}}=\chi} \mathcal{F}\left(T_{0}\right)_{\chi^{\prime}}
$$

For any $\alpha \in \Delta_{0}$ let $Z_{\alpha}=Z_{L_{\alpha}}$ where $L_{\alpha}$ is the Levi part of the maximal parabolic subgroup defined by $\alpha$. Explicitly,

$$
Z_{\alpha}=\bigcap_{\alpha \neq \beta \in \Delta_{0}} \operatorname{Ker} \beta .
$$


Also, if $P$ is a parabolic subgroup and $\alpha \in \Delta_{0} \backslash \Delta_{0}^{P}$, then we write $P^{\alpha}$ for the parabolic subgroup defined by

$$
\Delta_{0}^{P^{\alpha}}=\Delta_{0}^{P} \cup\{\alpha\}
$$

and $M^{\alpha}$ its Levi subgroup. Thus, $M$ is a co-rank one Levi subgroup of $M^{\alpha}$. Note that

$$
\mathbb{M}_{P^{\alpha}}=\left\{x \in \mathbb{M}_{P}: x_{\alpha} \neq 0\right\}
$$

and

$$
\mathcal{S}\left(\mathbb{M}_{P^{\alpha}}\right)=\left\{f \in \mathcal{S}\left(\mathbb{M}_{P}\right): f(x)=0 \text { if } x_{\alpha}=0\right\} .
$$

We have the following elementary properties.

Lemma 2.6. (1) For any $\chi \in \widehat{Z_{G}}, \mathfrak{F}_{G, \chi}$ is the space of smooth functions on $T_{0}$ which are compactly supported modulo $Z_{G}$ and have generalized eigenvalue $\chi$ under $Z_{G}$.

(2) For any $\phi \in \mathfrak{F}_{P, \chi}$ which is $T_{G}$-invariant there exist constants $C, n, k$ such that

$$
|\phi(t)| \leq C q^{-\langle\operatorname{Re} \chi, H(t)\rangle} \prod_{\alpha \in \Delta_{0}} \theta_{P, \alpha}^{n, k}(t), \quad t \in T_{0}
$$

where for any $\alpha \in \Delta_{0}$ and $t \in T_{0}$ we set

$$
\theta_{P, \alpha}^{n, k}(t)= \begin{cases}1 & \text { if } \alpha \in \Delta_{0}^{P} \text { and }|v(\alpha(t))| \leq n, \\ (v(\alpha(t))+n+1)^{k} & \text { if } \alpha \notin \Delta_{0}^{P} \text { and } v(\alpha(t)) \geq-n, \\ 0 & \text { otherwise. }\end{cases}
$$

(3) Suppose that $P^{\prime} \subseteq P$ and $\chi^{\prime} \in \widehat{Z_{M^{\prime}}}$ with $\left.\chi^{\prime}\right|_{Z_{M}}=\chi$. Then

$$
\mathfrak{F}_{P, \chi} \subseteq \mathfrak{F}_{P^{\prime}, \chi^{\prime}}
$$

(4) For $t \in T_{0}$ set

$$
D_{t_{0}} \phi(t)=\phi\left(t t_{0}\right)-\chi\left(t_{0}\right) \phi(t) .
$$

Then for any $\alpha \in \Delta_{0} \backslash \Delta_{0}^{P}, t_{0} \in Z_{\alpha}, \chi \in \widehat{Z_{M}}$ and $n>0$ we have

$$
D_{t_{0}} \mathfrak{F}_{P, \chi, n} \subseteq \mathfrak{F}_{P, \chi, n-1}+\mathfrak{F}_{P^{\alpha},\left.\chi\right|_{M^{\alpha}}, n} .
$$

(5) Suppose that $\phi_{i} \in \mathfrak{F}_{P_{i}, \chi_{i}}, i=1,2$. Then

$$
\phi_{1} \phi_{2} \in \mathfrak{F}_{P_{1} \bullet P_{2},\left.\chi_{1} \chi_{2}\right|_{Z_{M_{1}} \bullet M_{2}}} .
$$

In particular, for any $\phi \in \mathfrak{F}_{P, \chi}$ and $\mu \in \widehat{T_{0}}$ we have

$$
\phi \mu \in \mathfrak{F}_{P,\left.\chi \mu\right|_{Z_{M}}} .
$$

Proof. Clearly, any $\phi \in \mathfrak{F}_{G, \chi}$ is compactly supported modulo $Z_{G}$ and has generalized eigenvalue $\chi$ under $Z_{G}$. Conversely, suppose that $\phi$ is smooth, compactly supported modulo $Z_{G}$ and has generalized eigenvalue $\chi$ under $Z_{G}$. Replacing $\phi$ by $\chi^{-1} \phi$ we can assume that $\chi$ is trivial. By decomposing the support of $\phi$ to finitely many cosets of $Z_{G}$ it is enough to consider the case where $\phi$ is given by

$$
\phi(t)= \begin{cases}f(z) & \text { if } t=z v t_{0}, \quad z \in Z_{G}, v \in V \\ 0 & \text { otherwise }\end{cases}
$$


where $f \in \mathcal{F}\left(Z_{G}\right)_{1}, V$ is a compact open subgroup of $T_{0}^{1}$ and $t_{0} \in T_{0}$. The function $f$ is a polynomial function on the lattice $Z_{G} / Z_{G}^{1}$. It can be extended to a polynomial on the lattice $T_{0} / T_{0}^{1}$, i.e., to a function $\xi \in \mathcal{F}\left(T_{0}\right)_{1}$. By (2.12) we may write

$$
\phi(t)=\xi\left(t t_{0}^{-1}\right) \mathbf{1}_{\mathfrak{r}\left(V t_{0}\right)}(\mathfrak{r}(t)) .
$$

Thus, $\phi \in \mathfrak{F}_{G, \chi}$ as required.

For the second part we note that any $\phi \in \mathfrak{F}_{P, \chi}$ is compactly supported modulo $T_{M}$. Therefore, it is enough to check (2.15) on any fixed coset of $T_{M} \cap T_{0}^{G}$. This follows from the description (2.10).

For the third part, let $\phi \in \mathfrak{F}_{P, \chi}$. To show that $\phi \in \mathfrak{F}_{P^{\prime}, \chi^{\prime}}$ we can assume by (2.13) that

$$
\phi(t)=\xi(t) \varphi(\mathfrak{r}(t))
$$

with $\varphi \in \mathcal{S}\left(\mathbb{M}_{P}\right)$ and $\xi \in \mathcal{F}\left(T_{0}\right)_{\tilde{\chi}}$ where $\tilde{\chi} \in \widehat{T_{0}}$ and $\left.\tilde{\chi}\right|_{Z_{M}}=\chi$. Let $\tilde{\chi}^{\prime} \in \widehat{T_{0}}$ be an arbitrary extension of $\chi^{\prime}$. Then

$$
\eta:=\tilde{\chi}^{\prime} \tilde{\chi}^{-1} \in \widehat{T_{0}}
$$

is trivial on $Z_{M}$. Using (2.1) we can write

$$
\eta(t)^{-1}=\tilde{\varphi}(\mathfrak{r}(t))
$$

where $\tilde{\varphi}$ is a smooth function on $\mathbb{M}_{G}$ depending only on the coordinates in $\Delta_{0}^{P}$. Thus, we have

where

$$
\phi(t)=\xi^{\prime}(t) \varphi^{\prime}(\mathfrak{r}(t))
$$

and

$$
\xi^{\prime}=\xi \eta \in \mathcal{F}\left(T_{0}\right)_{\tilde{\chi}^{\prime}}
$$

$$
\varphi^{\prime}=\varphi \tilde{\varphi} \in \mathcal{S}\left(\mathbb{M}_{P}\right) \subseteq \mathcal{S}\left(\mathbb{M}_{P^{\prime}}\right) .
$$

To show the fourth part, suppose that $\phi(t)=\xi(t) \varphi(\mathfrak{r}(t))$ where $\xi \in \mathcal{F}\left(T_{0}\right)_{\chi}$ and $\varphi \in \mathcal{S}\left(\mathbb{M}_{P}\right)$. Then

$$
D_{t_{0}} \phi(t)=\left[\xi\left(t t_{0}\right)-\chi\left(t_{0}\right) \xi(t)\right] \varphi\left(\mathfrak{r}(t) \mathfrak{r}\left(t_{0}\right)\right)+\chi\left(t_{0}\right) \xi(t)\left[\varphi\left(\mathfrak{r}(t) \mathfrak{r}\left(t_{0}\right)\right)-\varphi(\mathfrak{r}(t))\right] .
$$

Since $\mathfrak{r}\left(t_{0}\right)$ is 1 in all coordinates except $\alpha$, we have

$$
\varphi\left(\cdot \mathfrak{r}\left(t_{0}\right)\right)-\varphi(\cdot) \in \mathcal{S}\left(\mathbb{M}_{P^{\alpha}}\right)
$$

by (2.14).

Finally, the last part follows from (2.9), (2.11) and (2.13).

Remark 2.7. The definition of $\mathfrak{F}_{P, \chi}$ is tailor-made for split groups. In order to deal with general quasi-split groups we have to modify the definition of $\mathfrak{F}_{P, \chi}$ to a space of functions on $C_{G}\left(T_{0}\right)$ (where $T_{0}$ is a maximal split torus). One has to take into account that the simple roots of $T_{0}$ do not necessarily extend to $C_{G}\left(T_{0}\right)$.

For any $\alpha \in \Delta_{0}$ the lattice $T_{G} Z_{\alpha}^{1} \backslash Z_{\alpha} \simeq X_{*}\left(T_{G} \backslash Z_{\alpha}\right)$ is one-dimensional. We fix an element $t_{\alpha} \in Z_{\alpha}$ which lies above a generator of $T_{G} Z_{\alpha}^{1} \backslash Z_{\alpha}$ and such that $\left|\alpha\left(t_{\alpha}\right)\right|<1$. We also fix a generating set $\omega_{1}^{\vee}, \ldots, \omega_{d}^{\vee}$ for $X_{*}\left(T_{G} \backslash T_{0}\right)$.

For any $\chi \in \widehat{Z_{M}}$ we set

$$
\mathfrak{S}(\chi)=\left\{\alpha \in \Delta_{0} \backslash \Delta_{0}^{P}:\left.\chi\right|_{Z_{\alpha}^{1}} \equiv 1\right\} .
$$

Note that if $P^{\prime} \subseteq P$ and $\chi^{\prime} \in \widehat{Z_{M^{\prime}}}$, then

$$
\mathfrak{S}\left(\left.\chi^{\prime}\right|_{Z_{M}}\right)=\mathfrak{S}\left(\chi^{\prime}\right) \backslash \Delta_{0}^{P} .
$$


Lemma 2.8. Let $\phi \in \mathfrak{F}_{P, \chi}$ and suppose that $\phi$ is $T_{G}$-invariant. (In particular, $\left.\chi\right|_{T_{G}} \equiv 1$.) Then:

(1) The integral

$$
I(\phi, \lambda)=\int_{T_{G} \backslash T_{0}} \phi(t) q^{-\langle\lambda, H(t)\rangle} d t
$$

converges in the cone

$$
\left\{\lambda \in\left(\mathfrak{a}_{0}^{G}\right)_{\mathbb{C}}^{*}:\left\langle\operatorname{Re} \lambda+\operatorname{Re} \chi, \varpi^{\vee}\right\rangle>0 \text { for all } \varpi^{\vee} \in \hat{\Delta}_{P}^{\vee}\right\}
$$

and defines a rational function in $q^{\left\langle\lambda, \omega_{i}^{\vee}\right\rangle}, i=1, \ldots, d$ with poles at most along the hyperplanes

$$
q^{\left\langle\lambda, H\left(t_{\alpha}\right)\right\rangle}=\chi\left(t_{\alpha}\right), \quad \alpha \in \mathfrak{S}(\chi) .
$$

(2) Let $\omega=\sum_{\alpha \in \Delta_{0}} n_{\alpha} \alpha \in X^{*}\left(T_{0}\right)$ with $n_{\alpha}>0$. Define

$$
a_{n}=a_{n}(\phi)=\int_{T_{G} \backslash T_{0}} \delta_{\langle\omega, H(t)\rangle, n} \phi(t) d t
$$

where $\delta_{m, n}$ is the Kronecker delta. Then $a_{n}$ is e.p.e. and

$$
\operatorname{Re} \mathcal{E}\left(\left(a_{n}\right)\right) \subseteq \bigcup_{\alpha \in \mathfrak{S}(\chi)}\left\{\lambda:\left|\omega\left(t_{\alpha}\right)\right|^{-\lambda}=\left|\chi\left(t_{\alpha}\right)\right|\right\} .
$$

Proof. For the convergence part we use (2.15) to reduce it to the convergence of

$$
\int_{T_{G} \backslash T_{0}} q^{\langle-(\operatorname{Re} \lambda+\operatorname{Re} \chi), H(t)\rangle} \prod_{\alpha \in \Delta_{0}} \theta_{P, \alpha}^{m, k}(t) d t .
$$

The integrand is clearly invariant under $T_{0}^{1}$. By writing

$$
H(t)=\sum_{\alpha \in \Delta_{0}} v(\alpha(t)) \varpi_{\alpha}^{\vee} \bmod \mathfrak{a}_{G}
$$

the convergence further reduces to that of

$$
\sum_{n \geq-m} q^{-n\left(\left\langle\operatorname{Re} \lambda+\operatorname{Re} \chi, \varpi^{\vee}\right\rangle\right)}(1+|n|)^{k}
$$

for all $\varpi^{\vee} \in \hat{\Delta}_{P}^{\vee}$.

Similarly, the integrand in the definition of $a_{n}$ is compactly supported in the domain of integration. Therefore, $a_{n}$ is well defined and it is easy to see that $a_{n}=0$ for $n \ll 0$.

Suppose that $\phi \in \mathfrak{F}_{P, \chi, m}$. We will prove the remaining two statements by induction on $m$ and $r$ where $r$ is the co-rank of $P$. If $m=0$, then $\phi=0$ and there is nothing to prove. If $r=0$, then $P=G$ and $\phi \in \mathcal{S}\left(T_{G} \backslash T_{0}\right)$. In this case $a_{n}=0$ for almost all $n$ and $I(\phi, \lambda)$ reduces to a polynomial in $q^{ \pm\left\langle\lambda, \omega_{i}^{\vee}\right\rangle}$.

For the induction step, suppose that $P \neq G$ and $m>0$. Let $\alpha \in \Delta_{0} \backslash \Delta_{0}^{P}$. For any $t_{0} \in Z_{\alpha}$ we have

$$
\begin{gathered}
I\left(D_{t_{0}} \phi, \lambda\right)=\left(q^{\left\langle\lambda, H\left(t_{0}\right)\right\rangle}-\chi\left(t_{0}\right)\right) I(\phi, \lambda), \\
a_{n}\left(D_{t_{0}} \phi\right)=a_{n+v\left(\omega\left(t_{0}\right)\right)}(\phi)-\chi\left(t_{0}\right) a_{n}(\phi) .
\end{gathered}
$$

If $\alpha \notin \mathfrak{S}(\chi)$, we take $t_{0} \in Z_{\alpha}^{1}$ such that $\chi\left(t_{0}\right) \neq 1$. Otherwise, take $t_{0}=t_{\alpha}$. In the first case $H\left(t_{0}\right) \in \mathfrak{a}_{G}$ and thus $q^{\left\langle\lambda, H\left(t_{0}\right)\right\rangle}=1$. In both cases, the lemma follows from (2.16) and Corollary 2.4 by applying the induction hypothesis to $D_{t_{0}} \phi$. 
2.5. Germs. For any parabolic subgroup $P=M U$ and $\epsilon>0$ let

$$
M_{<\epsilon}=\left\{m \in M:|\alpha|(m)<\epsilon, \quad \forall \alpha \in \Delta_{P}\right\} .
$$

We set an equivalence relation on $C^{\infty}(M)$ by saying that $f_{1}$ and $f_{2}$ have the same germ (at 0 ) if they agree on $M_{<\epsilon}$ for some $\epsilon>0$. This equivalence relation clearly respects the left and right action of $M$ and therefore the space $\mathcal{G}(M)$ of equivalence classes is also a representation space of $M$. Similarly, we can define the set $\left(Z_{M}\right)_{<\epsilon}$ and the space $\mathcal{G}\left(Z_{M}\right)$ of germs of functions on $Z_{M}$.

Lemma 2.9. The map $f \mapsto[f]$ sending $f$ to its germ (i.e., its equivalence class) induces an isomorphism of $Z_{M}$-modules

$$
\Gamma: \mathcal{F}\left(Z_{M}\right) \rightarrow \mathcal{G}\left(Z_{M}\right)_{Z_{M} \text {-fin }} .
$$

Moreover, there exists $\delta>0$ with the following property. Let $V$ be an open subgroup of $Z_{M}^{1}, \chi \in \widehat{Z_{M} / V}, n \in \mathbb{N}$ and $\mathcal{B} \subseteq Z_{M}$ a finite set such that $\mathcal{B} V$ generates $Z_{M}$. Assume that $\mathcal{B}$ contains $t_{\alpha}, \alpha \in \Delta_{0} \backslash \Delta_{0}^{P}$ as well as a set of elements $z_{1}, \ldots, z_{s} \in T_{G}$ such that $H\left(z_{i}\right), i=1, \ldots, s$ form a basis for $\mathfrak{a}_{G}$. Suppose that $f \in C^{\infty}\left(Z_{M}\right)$ is $V$-invariant and

$$
\prod_{i=1}^{n}\left(R\left(b_{i}\right)-\chi\left(b_{i}\right)\right) f(t)=0
$$

for all $t \in\left(Z_{M}\right)_{<\epsilon}$ and $b_{1}, \ldots, b_{n} \in \mathcal{B}$. Then

$$
f(t)=\Gamma^{-1}([f])(t)
$$

for all $t \in\left(Z_{M}\right)_{<\epsilon \delta}$.

For the proof we will use the following well-known fact whose elementary proof will be omitted.

Lemma 2.10. Let $r, s \in \mathbb{N}$ and let $X$ be the monoid $\mathbb{N}^{r} \times \mathbb{Z}^{s}$ with the standard generators $e_{1}, \ldots, e_{r}, \pm e_{r+1}, \ldots, \pm e_{r+s}$. Let $\varphi$ be a function on $X$. Suppose that for any $i=1, \ldots, r+s$ the function $\varphi\left(e_{i}+x\right)-\varphi(x)$ is a polynomial on $X$. Then $\varphi$ is a polynomial on $X$.

Proof of Lemma 2.9. The injectivity of $\Gamma$ reduces to the corresponding statement for $T_{M}$, which in turn follows from the description (2.10) of $\mathcal{F}\left(T_{M}\right)$.

To show surjectivity and the last part, let $f \in C^{\infty}\left(Z_{M}\right)$ be such that $[f] \in$ $\mathcal{G}\left(Z_{M}\right)_{\chi, n}$. Thus, there exists $\epsilon>0$ such that

$$
\prod_{i=1}^{n}\left(R\left(b_{i}\right)-\chi\left(b_{i}\right)\right) f(t)=0
$$

for all $b_{i} \in \mathcal{B}$ and $t \in\left(Z_{M}\right)_{<\epsilon}$. We will show that for an appropriate $\delta>0$ (independent of $f$ and $\epsilon$ ) there exists $g \in \mathcal{F}\left(Z_{M}\right)$ such that

$$
g \equiv f \text { on }\left(Z_{M}\right)_{<\epsilon \delta} .
$$

Replacing $f$ by $f \chi^{-1}$, we can assume that $\chi$ is trivial. Observe that $f$ is $Z_{M^{-}}^{1}$ invariant on $\left(Z_{M}\right)_{<\epsilon}$, since $Z_{M}^{1}$ is compact. Thus, the restriction of $f$ to $\left(Z_{M}\right)_{<\epsilon}$ depends only on $H(t)$. Let $X$ (resp. $\tilde{X}$ ) be the monoid (resp. group) generated by $t_{\alpha}, \alpha \in \Delta_{0} \backslash \Delta_{0}^{P}$ and $z_{i}^{ \pm 1}, i=1, \ldots, s$. Note that $\tilde{X}$ is free. Fix a finite set of representatives $\mathcal{Y}$ for $Z_{M} / Z_{M}^{1} \tilde{X}$. We can choose $\mathcal{Y}$ so that

$$
\epsilon \delta<|\alpha|(y)<\epsilon, \quad y \in \mathcal{Y}, \alpha \in \Delta_{0} \backslash \Delta_{0}^{P}
$$


where $\delta$ is a uniform constant. Thus,

$$
\left(Z_{M}\right)_{<\epsilon \delta} \subseteq Z_{M}^{1} X \mathcal{Y} \subseteq\left(Z_{M}\right)_{<\epsilon}
$$

We will show that for any $y \in \mathcal{Y}$ there exists a polynomial $Q_{y}$ on $\mathfrak{a}_{M}$ such that

$$
f(t y)=Q_{y}(H(t)) \text { for all } t \in X .
$$

We use induction on $n$. The statement is clear for $n=0$. For the induction step, we know that for each $y \in \mathcal{Y}$ there exist polynomials $Q_{y, \alpha}, \alpha \in \Delta_{0} \backslash \Delta_{0}^{P}$ and $Q_{y, i}^{\prime}$, $i=1, \ldots, s$ such that for all $t \in X$ we have

$$
\begin{gathered}
f\left(t_{\alpha} t y\right)-f(t y)=Q_{y, \alpha}(H(t)) \quad \alpha \in \Delta_{0} \backslash \Delta_{0}^{P}, \\
f\left(z_{i} t y\right)-f(t y)=Q_{y, i}^{\prime}(H(t)) \quad i=1, \ldots, s .
\end{gathered}
$$

Our claim now follows from Lemma 2.10 .

Thus, the function $g$ on $Z_{M}$ defined by

$$
g(a t y)=Q_{y}(H(t)) \quad y \in \mathcal{Y}, t \in \tilde{X}, a \in Z_{M}^{1}
$$

is $Z_{M}$-finite and agrees with $f$ on $\left(Z_{M}\right)_{<\epsilon \delta}$.

Corollary 2.11. The map $f \mapsto[f]$ induces a bi-M-equivariant isomorphism

$$
\iota_{M}: C^{\infty}(M)_{Z_{M} \text {-fin }} \rightarrow \mathcal{G}(M)_{Z_{M} \text {-fin }}
$$

Proof. Let $f \in C^{\infty}(M)$ and for each $m \in M$ let $f_{m}(t)=f(t m), t \in Z_{M}$. Suppose that $f$ is $Z_{M}$-finite and $\iota_{M}(f)=0$. Then for any $m \in M, f_{m} \in \mathcal{F}\left(Z_{M}\right)$ and $\Gamma\left(f_{m}\right)=0$. By the lemma we conclude that $f_{m} \equiv 0$ and therefore $f \equiv 0$. Thus, $\iota_{M}$ is injective.

To show surjectivity, suppose that $f \in C^{\infty}(M)$ and $[f] \in \mathcal{G}(M)_{\chi, n}$ for some $\chi \in \widehat{Z_{M}}$ and $n$. Then $\left[f_{m}\right] \in \mathcal{G}\left(Z_{M}\right)_{\chi, n}$ for all $m \in M$. Define

$$
\tilde{f}(m)=\Gamma^{-1}\left(\left[f_{m}\right]\right)(1) .
$$

Then $\tilde{f} \in C^{\infty}(M)$, since for any $m_{0}$ in a small neighborhood of 1 we have $f_{m}=$ $f_{m m_{0}}$ as $f \in C^{\infty}(M)$. Since $\Gamma$ is $Z_{M^{-} \text {-equivariant, we have }}$

$$
\tilde{f}(t m)=\Gamma^{-1}\left(\left[f_{m}\right]\right)(t)
$$

for all $t \in Z_{M}$. Therefore,

$$
\tilde{f}_{m} \in \mathcal{F}\left(Z_{M}\right)_{\chi, n}
$$

for all $m \in M$. Thus,

$$
\tilde{f} \in C^{\infty}(M)_{\chi, n}
$$

Finally, by the last part of the Lemma there exists $\epsilon>0$ such that

$$
\tilde{f}(m)=f_{m}(1)=f(m)
$$

for all $m \in M_{<\epsilon}$. It follows that $\iota_{M}(\tilde{f})=[f]$ as required. 


\section{The MAIN RESULT}

Let $\pi$ be a smooth representation of $G$. For any parabolic subgroup $P \supseteq B$ let $J_{P}(\pi)$ denote the Jacquet module of $\pi$ with respect to $P$, viewed as a smooth representation of $M$. Let $\mathcal{E}_{P}(\pi)$ denote the set of cuspidal exponents of $\pi$ along $P$, i.e., those $\chi \in \widehat{Z_{M}}$ such that $J_{P}(\pi)_{\chi}$, the $\chi$-generalized eigenspace of $J_{P}(\pi)$, contains a supercuspidal constituent. Set

$$
\mathcal{E}(\pi)=\bigcup_{P} \mathcal{E}_{P}(\pi) \neq \emptyset
$$

If $\pi$ is of finite length, then $\mathcal{E}(\pi)$ is finite. If $\pi$ is irreducible, then there exists $P$ such that $\mathcal{E}_{Q}(\pi)=\emptyset$ unless $Q$ and $P$ are associated.

Fix a non-degenerated character $\psi: U_{0} \rightarrow \mathbb{C}^{*}$ of $U_{0}$, that is, $\left.\psi\right|_{U_{\alpha}} \not \equiv 1$ for all $\alpha \in \Delta_{0}$. Let $\Omega(G)$ be the $G$-space of smooth function $W: G \rightarrow \mathbb{C}$ such that $W(u g)=\psi(u) W(g)$ for all $u \in U_{0}, g \in G$, with $G$ acting by right translation.

Suppose that $\pi$ is an irreducible generic representation of $G$. That is, $\pi$ can be realized as a subspace $\mathcal{W}(\pi)$ of $\Omega(G)$. The space $\mathcal{W}(\pi)$ is uniquely determined by the equivalence class of $\pi$ and is called the Whittaker model of $\pi$. (Cf. [Sha74], GK75], BZ76], Rod73].)

Theorem 3.1. Let $(\pi, \mathcal{W}(\pi))$ be a subrepresentation of $\Omega(G)$ of finite length. Then there exists $n$ such that any $W \in \mathcal{W}(\pi)$ can be written as

$$
W(u t k)=\psi(u) \sum_{P \supseteq B} \delta_{P}^{\frac{1}{2}}(t) \sum_{\chi \in \mathcal{E}_{P}(\pi)} \phi_{P, \chi}(t, k) \quad t \in T_{0}, u \in U_{0}, k \in K
$$

where $\phi_{P, \chi}(\cdot, k) \in \mathfrak{F}_{P, \chi, n}$ for all $k \in K$ and $\phi_{P, \chi}$ is invariant under an open subgroup of $K$.

Proof. We will prove the theorem by induction on the semi-simple rank of $G$, the case where $G$ is a torus being trivial. Of course, we are primarily interested in irreducible representations. However, we need the finite length assumption to make the induction work.

First note that by considering finitely many translates of $W$ it is enough to prove the statement for $k$ being the identity element. Consider the map

$$
W \in \mathcal{W}(\pi) \mapsto\left[\left.\delta_{P}^{-\frac{1}{2}} W\right|_{M}\right] .
$$

We claim that it factors through the Jacquet module $J_{P}(\pi)$ and gives rise to an intertwining map

$$
\kappa_{M}: J_{P}(\pi) \rightarrow \mathcal{G}(M)
$$

Indeed, let $u \in U$. Then for $m \in M_{<\epsilon}$ for $\epsilon$ sufficiently small we have

$$
W(m u)=W\left(m^{-1} m\right)=\psi\left(m^{-1}\right) W(m)=W(m)
$$

so that the germs of $W$ and $\pi(u) W$ coincide. The equivariance property is clear (because of the $\delta_{P}^{\frac{1}{2}}$-shift in the definition of the Jacquet functor).

Since $J_{P}(\pi)$ is of finite length, all vectors in $J_{P}(\pi)$ and the image of $\kappa_{M}$ are $Z_{M}$-finite. Therefore, we get an $M$-equivariant map

$$
\Xi_{M}:=\iota_{M}^{-1} \circ \kappa_{M}: J_{P}(\pi) \rightarrow C^{\infty}(M)_{Z_{M} \text {-fin }}
$$

where $\iota_{M}$ is as in Corollary 2.11. Clearly, the image lies in the space $\Omega(M)$ of Whittaker functions on $M$. 
Next, observe that $W(t)=0$ if $|\alpha(t)| \gg 1$ for some $\alpha \in \Delta_{0}$. (Cf. [JPSS81, p. 204] for the $G L_{n}$ case.) Indeed, fix $u \in U_{\alpha}$ so that $\psi(u) \neq 1$. By the property of $t$, $u^{\prime}=t^{-1} u t$ is very close to 1 , and therefore $W$ is right invariant under $u^{\prime}$. Hence,

$$
W(t)=W\left(t u^{\prime}\right)=W(u t)=\psi(u) W(t)
$$

so that $W(t)=0$ as required.

We are now ready for the induction step. By passing to a direct summand we may assume that $\mathcal{W}(\pi)=\mathcal{W}(\pi)_{\mu}$ for some $\mu \in \widehat{Z_{G}}$. For any $\emptyset \neq I \subseteq \Delta_{0}$ let $P_{I}=M_{I} U_{I}$ be the proper parabolic subgroup of $G$ such that $\Delta_{0} \backslash \Delta_{0}^{P_{I}}=I$. Let $j_{I}: \pi \rightarrow J_{P_{I}}(\pi)$ be the canonical projection. By the transitivity of the Jacquet functor and the induction hypothesis applied to $J_{P_{I}}(\pi)$ we can write

$$
\left[\Xi_{M_{I}} j_{I}(W)\right](t)=\sum_{P \subseteq P_{I}} \delta_{P \cap M_{I}}^{\frac{1}{2}}(t) \sum_{\chi \in \mathcal{E}_{P}(\pi)} \phi_{P, \chi}^{I}(t)
$$

where $\phi_{P, \chi}^{I} \in \mathfrak{F}_{P \cap M_{I}, \chi}^{M_{I}}$. In other words, there exists $\epsilon>0$ such that

$$
W(t)=\sum_{P \subseteq P_{I}} \delta_{P}^{\frac{1}{2}}(t) \sum_{\chi \in \mathcal{E}_{P}(\pi)} \phi_{P, \chi}^{I}(t)
$$

provided that $|\alpha|(t)<\epsilon$ for all $\alpha \in \Delta_{P_{I}}$. Note that both sides of (3.1) vanish if $|\alpha(t)|$ is large for some $\alpha \in \Delta_{0}^{P_{I}}$. Therefore, it follows from Remark 2.1 that for an appropriate $\epsilon>0$, (3.1) holds whenever $|\alpha(t)|<\epsilon$ for all $\alpha \in I$. Fix such $\epsilon$ which works for all $I \neq \emptyset$. Set

$$
\phi_{P, \chi}(t)=\sum_{\emptyset \neq I \subseteq \Delta_{0} \backslash \Delta_{0}^{P}}(-1)^{|I|-1} \phi_{P, \chi}^{I}(t) \prod_{\alpha \in I} \mathbf{1}_{<\epsilon}(|\alpha(t)|)
$$

where $\mathbf{1}_{<\epsilon}$ denotes the characteristic function of $(0, \epsilon)$. Then $\phi_{P, \chi} \in \mathfrak{F}_{P, \chi}$ and

$$
\sum_{P \subsetneq G} \sum_{\chi \in \mathcal{E}_{P}(\pi)} \delta_{P}^{\frac{1}{2}}(t) \phi_{P, \chi}(t)=W(t) \sum_{\emptyset \neq I \subseteq \Delta_{0}}(-1)^{|I|-1} \prod_{\alpha \in I} \mathbf{1}_{<\epsilon}(|\alpha(t)|) .
$$

By the inclusion-exclusion principle we get

$$
\sum_{P \subsetneq G} \sum_{\chi \in \mathcal{E}_{P}(\pi)} \delta_{P}^{\frac{1}{2}}(t) \phi_{P, \chi}(t)= \begin{cases}W(t) & \text { if }|\alpha(t)|<\epsilon \text { for some } \alpha \in \Delta_{0}, \\ 0 & \text { otherwise. }\end{cases}
$$

Let $Q$ be such that $\mathcal{E}_{Q}(\pi) \neq \emptyset$ and take any $\omega \in \mathcal{E}_{Q}(\pi)$. Then $\left.\omega\right|_{Z_{G}}=\mu$ and therefore

$$
\phi^{\prime}(t):=\delta_{Q}^{-\frac{1}{2}}(t) W(t) \prod_{\alpha \in \Delta_{0}} \mathbf{1}_{\geq \epsilon}(|\alpha(t)|) \in \mathfrak{F}_{G, \mu} \subseteq \mathfrak{F}_{Q, \omega}
$$

by Lemma 2.6. The conclusion of the theorem holds upon replacing $\phi_{Q, \omega}$ by $\phi_{Q, \omega}+$ $\phi^{\prime}$.

The theorem just proved shows that the asymptotic behavior of the Whittaker functions are completely governed by the exponents of the representation. It is natural to ask to what extent the converse is true. Namely,

Question 3.2. What is the kernel of the maps $\kappa_{M}$ ? 
Suppose that $\pi$ is irreducible, generic and has a unitary central character. For $W_{1}, W_{2} \in \mathcal{W}(\pi)$ and $\lambda \in\left(\mathfrak{a}_{0}^{G}\right)_{\mathbb{C}}^{*}$, define

$$
I\left(W_{1}, W_{2}, \lambda\right)=\int_{T_{G} U_{0} \backslash G} q^{-\langle\lambda, H(g)\rangle} W_{1}(g) \overline{W_{2}(g)} d g .
$$

Corollary 3.3. The integral $I\left(W_{1}, W_{2}, \lambda\right)$ is absolutely convergent whenever

$$
\left\langle\operatorname{Re} \lambda+2 \operatorname{Re} \chi, \varpi^{\vee}\right\rangle>0
$$

for all $P, \varpi^{\vee} \in \hat{\Delta}_{P}^{\vee}$ and $\chi \in \mathcal{E}_{P}(\pi)$. It extends to a rational function in $q^{\left\langle\lambda, \omega_{i}^{\vee}\right\rangle}$, $i=1, \ldots, d$ with poles at most along

$$
q^{\left\langle\lambda, H\left(t_{\alpha}\right)\right\rangle}=\chi_{1} \overline{\chi_{2}}\left(t_{\alpha}\right)
$$

where $\chi_{i} \in \mathcal{E}_{P_{i}}(\pi), i=1,2$ and $\alpha \in \Delta_{0} \backslash\left(\Delta_{0}^{P_{1}} \cup \Delta_{0}^{P_{2}}\right)$ satisfy

$$
\chi_{1} \equiv \chi_{2} \text { on } Z_{\alpha}^{1} \text {. }
$$

Here $\omega_{1}^{\vee}, \ldots, \omega_{d}^{\vee}$ and $t_{\alpha}$ are as in Lemma 2.8 .

Proof. Denote the integrand of $I\left(W_{1}, W_{2}, \lambda\right)$ by $F(g)$. It is evidently left $T_{G} U_{0^{-}}$ invariant. We use the integration formula

$$
\int_{T_{G} U_{0} \backslash G} f(g) d g=\int_{K} \int_{T_{G} \backslash T_{0}} f(t k) \delta_{B}(t)^{-1} d t d k
$$

for any left $T_{G} U_{0}$-invariant continuous function on $G$. By Theorem 3.1, $F(t k)$ is a linear combination of functions of the form

$$
q^{-\langle\lambda, H(t)\rangle} \delta_{P_{1}}^{\frac{1}{2}}(t) \delta_{P_{2}}^{\frac{1}{2}}(t) \phi_{P_{1}, \chi_{1}}^{1}(t, k) \overline{\phi_{P_{2}, \chi_{2}}^{2}(t, k)}
$$

where $\phi_{P_{i}, \chi_{i}}^{i}(\cdot, k) \in \mathfrak{F}_{P_{i}, \chi_{i}}$ for $i=1,2$. Thus the integral $I\left(W_{1}, W_{2}, \lambda\right)$ is a finite sum of integrals of the form

$$
\int_{T_{G} \backslash T_{0}} q^{-\langle\lambda, H(t)\rangle} \delta_{P_{1}}^{\frac{1}{2}}(t) \delta_{P_{2}}^{\frac{1}{2}}(t) \phi_{1}(t) \overline{\phi_{2}(t)} \delta_{B}(t)^{-1} d t
$$

where $\phi_{i} \in \mathfrak{F}_{P_{i}, \chi_{i}}$ for $i=1,2$. From Lemma 2.6,

$$
\left.\phi_{1} \overline{\phi_{2}} \in \mathfrak{F}_{P_{1} \bullet P_{2}, \chi_{1} \overline{\chi_{2}}}\right|_{T_{M_{1} \bullet M_{2}}} .
$$

On the other hand, it follows from (2.2) that

$$
\delta_{P_{1}}^{\frac{1}{2}} \delta_{P_{2}}^{\frac{1}{2}} \equiv \delta_{B} \text { on } T_{M_{1} \bullet M_{2}}
$$

By (2.17) we infer that

$$
\left.\delta_{P_{1}}^{\frac{1}{2}} \delta_{P_{2}}^{\frac{1}{2}} \delta_{B}^{-1} \phi_{1} \overline{\phi_{2}} \in \mathfrak{F}_{P_{1} \bullet P_{2}, \chi_{1} \overline{\chi_{2}}}\right|_{T_{M_{1}} \bullet M_{2}}
$$

as well. We note that if

$$
\left\langle\operatorname{Re} \lambda+2 \operatorname{Re} \chi, \varpi^{\vee}\right\rangle>0
$$

for all $P, \varpi^{\vee} \in \hat{\Delta}_{P}^{\vee}$ and $\chi \in \mathcal{E}_{P}(\pi)$, then

$$
\left\langle\operatorname{Re} \lambda+\operatorname{Re} \chi_{1}+\operatorname{Re} \chi_{2}, \varpi^{\vee}\right\rangle>0
$$

for all $P_{1}, P_{2}, \varpi^{\vee} \in \hat{\Delta}_{P_{1}}^{\vee} \bullet P_{2}, \chi_{1} \in \mathcal{E}_{P_{1}}(\pi), \chi_{2} \in \mathcal{E}_{P_{2}}(\pi)$. The corollary now follows from Lemma 2.8 part 1 . 
Corollary 3.4. Suppose that $\pi$ is square-integrable and generic. Then the form

$$
\left(W_{1}, W_{2}\right)=\int_{T_{G} U_{0} \backslash G} W_{1}(g) \overline{W_{2}(g)} d g
$$

defines a non-zero $G$-invariant inner product on $\mathcal{W}(\pi)$.

Proof. The above integral, if convergent, is clearly $G$-invariant and positive-definite. We only need to check convergence. This follows from the previous corollary and the characterization of square-integrable representations by the positivity of their exponents, namely $\left\langle\operatorname{Re} \chi, \varpi^{\vee}\right\rangle>0$ for all $\varpi^{\vee} \in \hat{\Delta}_{P}^{\vee}$ and $\chi \in \mathcal{E}_{P}(\pi)$.

We conjecture the following converse to Corollary 3.4, which is related to Question 3.2 above.

Conjecture 3.5. Let $\pi$ be a generic representation, and suppose that

$$
\int_{T_{G} U_{0} \backslash G}|W(g)|^{2} d g<\infty
$$

for any $W \in \mathcal{W}(\pi)$. (It is enough to require this for a single $0 \neq W \in \mathcal{W}(\pi)$.) Then $\pi$ is square-integrable.

Finally, we extend Corollary 3.4 to the tempered case.

Corollary 3.6. Suppose that $\pi$ is tempered. Fix $\omega=\sum_{\alpha \in \Delta_{0}} n_{\alpha} \alpha \in X^{*}\left(T_{0}\right)$ with $n_{\alpha}>0$ for all $\alpha \in \Delta_{0}$. Then there exists $r=r(\pi, \omega) \in \mathbb{N}$ such that for any $W_{1}, W_{2} \in \mathcal{W}(\pi)$,

$$
\int_{g \in T_{G} U_{0} \backslash G:\langle\omega, H(g)\rangle \leq n} W_{1}(g) \overline{W_{2}(g)} d g \sim\left[W_{1}, W_{2}\right] n^{r} \quad \text { as } n \rightarrow \infty
$$

where $[\cdot, \cdot]$ is a non-zero invariant (positive-definite) inner product on $\mathcal{W}(\pi)$.

Proof. As before, it follows from Corollary 3.3 that $I\left(W_{1}, W_{2}, s \omega\right)$ is absolutely convergent for $\operatorname{Re}(s)>0$ and extends to a rational function in $q^{s}$. Let $r=r(W)$ be the order of the pole of $I(W, W, s \omega)$ at $s=0$. Fix $W \in \mathcal{W}(\pi)$ and write

$$
I(W, W, s \omega)=\sum a_{n} q^{-n s}
$$

for $\operatorname{Re} s>0$ where we set

$$
a_{n}=a_{n}(W):=\int_{T_{G} U_{0} \backslash G} \delta_{\langle\omega, H(g)\rangle, n}|W(g)|^{2} d g \geq 0 \quad n \in \mathbb{Z} .
$$

By Theorem 3.1 and Lemma 2.8, part 2 the sequence $a_{n}$ is e.p.e.. By Lemma 2.5 we have

$$
\int_{g \in T_{G} U_{0} \backslash G:\langle\omega, H(g)\rangle \leq n}|W(g)|^{2} d g \sim[W, W] n^{r(W)}
$$

as $n \rightarrow \infty$ where

$$
[W, W]:=\lim _{s \rightarrow 0} \frac{(s \log q)^{r(W)} I(W, W, s \omega)}{r(W) !} .
$$

Since

$$
a_{n}\left(W_{1}+W_{2}\right) \leq 2\left(a_{n}\left(W_{1}\right)+a_{n}\left(W_{2}\right)\right) \quad n \in \mathbb{Z}
$$

for all $W_{1}, W_{2} \in \mathcal{W}(\pi)$, it also follows from Lemma 2.5 that

$$
r\left(W_{1}+W_{2}\right) \leq \max \left(r\left(W_{1}\right), r\left(W_{2}\right)\right) .
$$


For $x \in G$, let $W_{x}(g)=W(g x)$. We show that

$$
\left[W_{x}, W_{x}\right]=[W, W] .
$$

It is a well-known fact that

$$
\{H(g)-H(g x): g \in G\}
$$

is a finite set (depending on $x$ ). Thus, there exists $C$ such that

$$
\begin{aligned}
& \left.\left|\int_{T_{G} U_{0} \backslash G} \mathbf{1}_{\leq n}(\langle\omega, H(g)\rangle)\right| W_{x}(g)\right|^{2} d g-\int_{T_{G} U_{0} \backslash G} \mathbf{1}_{\leq n}(\langle\omega, H(g)\rangle)|W(g)|^{2} d g \mid \\
& \quad=\left.\left|\int_{T_{G} U_{0} \backslash G} \mathbf{1}_{\leq n}\left(\left\langle\omega, H\left(g x^{-1}\right)\right\rangle\right)\right| W(g)\right|^{2} d g-\int_{T_{G} U_{0} \backslash G} \mathbf{1}_{\leq n}(\langle\omega, H(g)\rangle)|W(g)|^{2} d g \mid \\
& \quad \leq \sum_{|m-n| \leq C} a_{m} .
\end{aligned}
$$

By Lemma 2.5 the right-hand side is $O\left(n^{r(W)-1}\right)$ as $n \rightarrow \infty$. It follows once again from Lemma 2.5 that $r\left(W_{x}\right)=r(W)$ and

$$
\lim _{s \rightarrow 0} s^{r} I\left(W_{x}, W_{x}, s \omega\right)=\lim _{s \rightarrow 0} s^{r} I(W, W, s \omega) .
$$

Thus $\left[W_{x}, W_{x}\right]=[W, W]$. Moreover by irreducibility, $r=r(W)$ is independent of $W \neq 0$. Thus by polarization, $[\cdot, \cdot]$ defines a $G$-invariant form on $\mathcal{W}(\pi)$. The fact that $[\cdot, \cdot]$ is positive-definite follows from (3.2).

Example 3.7. Consider $G=P G L_{2}$. Let $\pi$ be the unramified tempered representation

$$
\operatorname{Ind}_{B}^{G}\left(\left(\begin{array}{ll}
a & 0 \\
0 & 1
\end{array}\right) \mapsto|a|^{\lambda}\right), \lambda \in \mathrm{i} \mathbb{R} .
$$

View $\psi$ as a character of $F$ and let $\mathfrak{c}(\psi)$ be the maximal fractional ideal on which $\psi$ is trivial. It is well known (cf. God70]) that the unramified Whittaker function normalized by $W(e)=1$ is given by

$$
|a|^{-\frac{1}{2}} W\left(\left(\begin{array}{cc}
a & 0 \\
0 & 1
\end{array}\right)\right)= \begin{cases}\frac{|a|^{\lambda}}{1-q^{2 \lambda}}+\frac{|a|^{-\lambda}}{1-q^{-2 \lambda}} & \text { if } a \in \mathfrak{c}(\psi) \text { and } \lambda \notin \frac{\pi \mathrm{i}}{\log q} \mathbb{Z}, \\
|a|^{\lambda}(1+v(a)) & \text { if } a \in \mathfrak{c}(\psi) \text { and } \lambda \in \frac{\pi \mathrm{i}}{\log q} \mathbb{Z}, \\
0 & \text { if } a \notin \mathfrak{c}(\psi) .\end{cases}
$$

It follows that for any positive $\omega, r=1$ if $\lambda \notin \frac{\pi \mathrm{i}}{\log q} \mathbb{Z}$ and $r=3$ otherwise.

In general, let $G$ be any split group and $\chi$ a regular unramified character of $T_{0}$, that is, $\chi^{w} \neq \chi$ for all $w \neq 1$ in the Weyl group of $G$. The representation $\operatorname{Ind}_{B}^{G} \chi$ is irreducible and it follows readily from the Casselman-Shalika formula for the unramified Whittaker function $(\mathrm{CS} 80])$, that $r\left(\operatorname{Ind}_{B}^{G} \chi, \omega\right)$ is the semi-simple rank of $G$ (regardless of $\omega$ ).

One can contemplate the following conjecture related to Conjecture 3.5 .

Conjecture 3.8. Suppose that $\pi$ is the generic constituent of $\operatorname{Ind}_{P}^{G} \tau$ where $\tau$ is a square-integrable generic representation of the Levi part of $P$. Suppose that the Plancherel measure on the component

$$
\operatorname{Ind}_{P}^{G} \tau q^{\langle\lambda, H(\cdot)\rangle}, \quad \lambda \in \mathrm{ia}_{M}^{*} / \frac{2 \pi \mathrm{i}}{\log q} X^{*}(M)
$$

is given by $\mu(\tau, \lambda) d \lambda$ where $\mu(\tau, \lambda)=\left|c_{\tau}(\lambda)\right|^{-2}$. Then $r(\pi, \omega)$ is equal to the sum of the co-rank of $P$ and the order of zero of $\mu(\tau, s \omega)$ at $s=0$. 
Remark 3.9. Let $\tilde{G}$ be the metaplectic cover of $S p_{2 n}$. One can define parabolic subgroups as the inverse images of parabolic subgroups of $S p_{2 n}$. The Jacquet functors are defined in an analogous way and satisfy the usual properties. In particular, they control the asymptotics of the matrix coefficients of the representation and the criterion for square-integrability is the same as for linear groups. Details will appear in a forthcoming paper of Szpruch. The notions of non-degenerate characters and generic representations are also defined and the uniqueness of Whittaker model is proved in [Szp07. The results of this paper as well as the proofs immediately carry over to $\tilde{G}$.

\section{REFERENCES}

[BZ76] I. N. Bernšteĭn and A. V. Zelevinskiŭ, Representations of the group $G L(n, F)$, where $F$ is a local non-Archimedean field, Uspehi Mat. Nauk 31 (1976), no. 3(189), 5-70. MR0425030 (54:12988)

[CPS] J. W. Cogdell and I. I. Piatetski-Shapiro, Derivatives and L-functions for $G L(n)$, To appear.

[CS80] W. Casselman and J. Shalika, The unramified principal series of p-adic groups. II. The Whittaker function, Compositio Math. 41 (1980), no. 2, 207-231. MR581582 (83i:22027)

[GK75] I. M. Gel'fand and D. A. Kajdan, Representations of the group $\operatorname{GL}(n, K)$ where $K$ is a local field, Lie groups and their representations (Proc. Summer School, Bolyai János Math. Soc., Budapest, 1971), Halsted, New York, 1975, pp. 95-118. MR0404534 (53:8334)

[God70] Roger Godement, Notes on Jacquet-Langlands' theory, (mimeographed notes).

[JPSS79] Hervé Jacquet, Ilja Iosifovitch Piatetski-Shapiro, and Joseph Shalika, Automorphic forms on GL(3). I, Ann. of Math. (2) 109 (1979), no. 1, 169-212. MR519356 (80i:10034a)

[JPSS81] H. Jacquet, I. I. Piatetski-Shapiro, and J. Shalika, Conducteur des représentations du groupe linéaire, Math. Ann. 256 (1981), no. 2, 199-214. MR620708 (83c:22025)

[JS90a] Hervé Jacquet and Joseph Shalika, Exterior square L-functions, Automorphic forms, Shimura varieties, and $L$-functions, Vol. II (Ann Arbor, MI, 1988), Perspect. Math., vol. 11, Academic Press, Boston, MA, 1990, pp. 143-226. MR1044830 (91g:11050)

[JS90b] L Rankin-Selberg convolutions: Archimedean theory, Festschrift in honor of I. I. Piatetski-Shapiro on the occasion of his sixtieth birthday, Part I (Ramat Aviv, 1989), Israel Math. Conf. Proc., vol. 2, Weizmann, Jerusalem, 1990, pp. 125-207. MR1159102 (93d:22022)

[Rod73] François Rodier, Whittaker models for admissible representations of reductive $p$-adic split groups, Harmonic analysis on homogeneous spaces (Proc. Sympos. Pure Math., Vol. XXVI, Williams College, Williamstown, MA, 1972), Amer. Math. Soc., Providence, RI, 1973, pp. 425-430. MR0354942 (50:7419)

[Sha74] J. A. Shalika, The multiplicity one theorem for $\mathrm{GL}_{n}$, Ann. of Math. (2) 100 (1974), 171-193. MR0348047 (50:545)

[Szp07] Dani Szpruch, Uniqueness of Whittaker model for the metaplectic group, Pacific J. Math. 232 (2007), no. 2, 453-469. MR2366363

[Wal92] Nolan R. Wallach, Real reductive groups. II, Pure and Applied Mathematics, vol. 132, Academic Press Inc., Boston, MA, 1992. MR1170566 (93m:22018)

Institute of Mathematics, Hebrew University of Jerusalem, Jerusalem Israel, 91904

E-mail address: erezla@math.huji.ac.il

Department of Mathematics and Computer Science, Rutgers University, Newark, NEW JERSEY 07102

E-mail address: zmao@rutgers.edu 